OPEN ACCESS

Edited by:

Aldo Corsetti,

Università di Teramo, Italy

Reviewed by: Maria Guadalupe Vizoso Pinto, National University of Tucumán,

Argentina

Cristian Botta

University of Turin, Italy

*Correspondence:

Seung Hyun Han

shhan-mi@snu.ac.kr

${ }^{\dagger}$ These authors have contributed equally to this work.

Specialty section:

This article was submitted to

Food Microbiology,

a section of the journal

Frontiers in Microbiology

Received: 24 July 2017 Accepted: 06 September 2017 Published: 21 September 2017

Citation:

Kim KW, Kang S-S, Woo S-J, Park O-J, Ahn KB, Song K-D, Lee H-K, Yun C-H and Han SH (2017) Lipoteichoic Acid of Probiotic Lactobacillus plantarum Attenuates

Poly I:C-Induced IL-8 Production in Porcine Intestinal Epithelial Cells.

Front. Microbiol. 8:1827.

doi: 10.3389/fmicb.2017.01827

\section{Lipoteichoic Acid of Probiotic Lactobacillus plantarum Attenuates Poly I:C-Induced IL-8 Production in Porcine Intestinal Epithelial Cells}

\author{
Kyoung Whun Kim ${ }^{1,2+}$, Seok-Seong Kang ${ }^{3 \dagger}$, Sun-Je Woo', Ok-Jin Park', Ki Bum Ahn', \\ Ki-Duk Song ${ }^{4}$, Hak-Kyo Lee ${ }^{4}$, Cheol-Heui Yun ${ }^{2,5}$ and Seung Hyun Han ${ }^{1 *}$ \\ 1 Department of Oral Microbiology and Immunology, DRI, and BK21 Plus Program, School of Dentistry, Seoul National \\ University, Seoul, South Korea, ${ }^{2}$ Department of Agricultural Biotechnology and Research Institute for Agriculture and Life \\ Sciences, Seoul National University, Seoul, South Korea, ${ }^{3}$ Department of Food Science and Biotechnology, Dongguk \\ University-Seoul, Seoul, South Korea, ${ }^{4}$ Department of Animal Biotechnology, Chonbuk National University, Jeonju, \\ South Korea, ${ }^{5}$ Institute of Green Bio Science Technology, Seoul National University, Seoul, South Korea
}

Probiotics in livestock feed supplements are considered a replacement for antibiotics that enhance gastrointestinal immunity. Although bacterial cell wall components have been proposed to be associated with probiotic function, little evidence demonstrates that they are responsible for probiotic functions in livestock. The present study demonstrated that lipoteichoic acid (LTA) of Lactobacillus plantarum (Lp.LTA) confers anti-inflammatory responses in porcine intestinal epithelial cell line, IPEC-J2. A synthetic analog of viral double-stranded RNA, poly I:C, dose-dependently induced IL-8 production at the mRNA and protein levels in IPEC-J2 cells. Lp.LTA, but not lipoprotein or peptidoglycan from L. plantarum, exclusively suppressed poly I:C-induced IL-8 production. Compared with LTAs from other probiotic Lactobacillus strains including L. delbrueckii, L. sakei, and L. rhamnosus GG, Lp.LTA had higher potential to suppress poly I:C-induced IL-8 production. Dealanylated or deacylated Lp.LTA did not suppress poly I:C-induced IL-8 production, suggesting that D-alanine and lipid moieties in the Lp.LTA structure were responsible for the inhibition. Furthermore, Lp.LTA attenuated the phosphorylation of ERK and p38 kinase as well as the activation of NF-kB, resulting in decreased IL-8 production. Taken together, these results suggest that Lp.LTA acts as an effector molecule to inhibit viral pathogen-induced inflammatory responses in porcine intestinal epithelial cells.

Keywords: probiotics, lipoteichoic acid, viral infection, inflammation, intestinal epithelial cells

\section{INTRODUCTION}

Probiotic bacteria are viable and defined microorganisms, which alter the composition and function of the intestinal microflora of humans and animals (Schrezenmeir and de Vrese, 2001). A set of major criteria for probiotics includes lack of transmissible antibiotic resistance genes, persistence of viability in the gastrointestinal tract, experimentally and clinically proven health benefits and safety for human and animal use (Lievin-Le Moal and Servin, 2014). Through production of metabolites including organic acids and bacteriocins, probiotic bacteria have 
beneficial effects on the intestines of mammals (O'Shea et al., 2012). The most extensively studied and widely used probiotic bacteria are Lactobacillus and Bifidobacterium (KoppHoolihan, 2001), which directly remove pathogens by producing antimicrobial compounds, decreasing $\mathrm{pH}$ by acids such as lactic acid, competing with pathogens for adhesion and colonization, as well as nutrients and other growth factors in the gut (Kailasapathy and Chin, 2000; Rolfe, 2000).

Increasing evidence suggests that probiotic bacteria regulate inflammation in a number of ways. For example, probiotic bacteria enhance the epithelial barrier function to prevent chronic inflammation in the gut (Bermudez-Brito et al., 2012). Certain probiotic strains inhibit pro-inflammatory cytokineimpaired epithelial integrity (Donato et al., 2010). By increasing the production of metabolites such as butyrate, probiotic bacteria suppress intestinal inflammatory responses (Thomas and Versalovic, 2010). Probiotic bacteria confer anti-inflammatory responses by modulating signaling pathways including nuclear factor (NF)- $\mathrm{B}$, mitogen-activated protein kinase (MAPK) and peroxisome proliferator-activated receptor gamma (PPAR $\gamma)$ (Thomas and Versalovic, 2010). In addition, probiotic lactobacilli down-regulate the production of interleukin (IL)-6 and IL-8 in intestinal epithelial cells (Villena and Kitazawa, 2014; Noh et al., 2015). Probiotic bacteria also exert inflammatory responses by interacting with Toll-like receptors (TLRs). TLR2 can be particularly important for regulating inflammatory signaling pathways for Gram-positive bacteria (Noh et al., 2015).

Although probiotic bacteria have a positive impact on certain infections, inflammatory reactions, and allergic diseases, clinically employed probiotics have been revealed as species-and strain-specific (Snel et al., 2011; Agustina et al., 2012). Several reports suggest that individual probiotic effector molecules communicating with hosts may underlie probiotic effects (Bron et al., 2013; Lee et al., 2013). Several effector molecules such as peptidoglycan (PGN) and lipoteichoic acid (LTA) are the major cell wall components of Gram-positive bacteria that can be considered the pivotal components for immunomodulating effects (Lee et al., 2013). In particular, the D-alanine moiety of LTA structure is considered an important functional group for immunostimulating effects (Morath et al., 2001). Moreover, lipid moieties of the LTA structure are also considered to be immunomodulating groups (Morath et al., 2002). LTA from pathogenic bacteria is responsible for the initiation and development of inflammatory responses (Kang et al., 2016). In contrast, Lactobacillus LTA is involved in anti-inflammatory responses (Grangette et al., 2005; Noh et al., 2015). Moreover, PGN derived from lactobacilli reduces inflammatory responses in the colon (Macho Fernandez et al., 2011), suggesting that the cell wall of probiotic lactobacilli contains potential immunomodulators.

Antibiotics have been used to prevent diarrhea and promote growth in farm animals including pigs (Thacker, 2013). However, because of increased negative effects including emerging bacterial resistance to antibiotics (van der Fels-Klerx et al., 2011), probiotic lactobacilli are suggested as an alternative strategy in swine production. Beneficial effects of probiotic lactobacilli such as regulation of intestinal microflora, prevention of intestinal pathogens and maintenance of intestinal barrier function (Yang et al., 2015) are suggested. Nevertheless, the cell wall-associated molecules of probiotic lactobacilli that attenuate gastrointestinal inflammatory reactivity have not yet been fully elucidated. In this study, we investigated the effects of probiotic Lactobacillus plantarum LTA to reduce IL- 8 production in porcine intestinal epithelial cells.

\section{MATERIALS AND METHODS}

\section{Bacteria and Reagents}

L. plantarum K8 (KCTC 10887BP) was obtained from the Korean Collection for Type Culture (Daejeon, South Korea). Poly I:C was purchased from InvivoGen (San Diego, CA, United States). Pam2CSK4 was purchased from EMC Microcollection GmbH (Tübingen, Germany). Antibodies specific to p38 kinase, phospho-p38 kinase, ERK, phospho-ERK, IкB $\alpha$, and $\beta$-actin were obtained from Cell Signaling Technology (Beverly, MA, United States).

\section{Preparation of Cell Wall Components of Lactobacillus}

L. plantarum LTA (Lp.LTA) was prepared and the absence of unwanted biological contaminants such as endotoxins, nucleic acids, and proteins was examined as described previously (Ryu et al., 2009). D-Alanine-deficient Lp.LTA (dealanylated) and acyl chain-deficient structure of Lp.LTA (deacylated) were prepared as described previously (Noh et al., 2015). LTAs from L. delbrueckii, L. sakei, and L. rhamnosus GG were kindly provided from Prof. Dae Kyun Chung (Kyung Hee University, Suwon, South Korea). Lipoproteins of L. plantarum were isolated as described previously (Kang et al., 2015). PGN from L. plantarum was prepared as described previously (Noh et al., 2015).

\section{Cell Culture}

A non-transformed IPEC-J2 cell line, originally isolated from jejunum of a neonatal piglet, was obtained from the American Type Culture Collection (Manassas, VA, United States), and grown in Dulbecco's Modified Eagle's Medium and Ham's F-12 media (1:1) (HyClone, Logan, UT, United States) supplemented with $10 \%$ fetal bovine serum (Gibco, Burlington, ON, Canada), $1 \%$ insulin-transferrin-selenium G supplement (Gibco), $100 \mathrm{U} / \mathrm{ml}$ penicillin, and $100 \mu \mathrm{g} / \mathrm{ml}$ streptomycin (HyClone) at $37^{\circ} \mathrm{C}$ in a $5 \% \mathrm{CO}_{2}$ atmosphere in a humidified incubator.

\section{Real-time Quantitative PCR}

After simultaneous treatment with indicated stimuli in IPEC-J2 cells for $3 \mathrm{~h}$, total RNA was extracted using TRIzol reagent (Invitrogen, Carlsbad, CA, United States) according to the manufacturer's instructions. Five micrograms of total RNA was subjected to complementary DNA (cDNA) synthesis using random hexamers and reverse transcriptase (Promega, Madison, WI, United States). Real-time quantitative PCR was performed with SYBR Premix II Ex Taq (Takara Bio, Shiga, Japan) using an 
ABI Prism 7500 Sequence Detection System (Applied Biosystems, Foster City, CA, United States). Amplification of cDNA was performed as follows: denaturation for $10 \mathrm{~s}$ at $95^{\circ} \mathrm{C}$ and amplification for 40 cycles of $5 \mathrm{~s}$ at $95^{\circ} \mathrm{C}$ and for $34 \mathrm{~s}$ at $60^{\circ} \mathrm{C}$. The $\Delta \mathrm{Ct}$ was obtained by subtracting the $\mathrm{Ct}$ value of $\beta$-actin gene from the Ct value of IL- 8 gene and $\Delta \mathrm{Ct}$ of the non-treatment control group was used as the calibrator. The

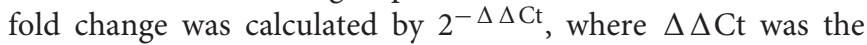
difference between $\Delta \mathrm{Ct}$ and the $\Delta \mathrm{Ct}$ calibrator value, which was assigned a value of 1 arbitrary unit. Sequences of specific primers for IL- 8 and $\beta$-actin were as follows: IL- 8 sense primer, $5^{\prime}$-CCTGCTTTCTGCAGCTCTCT-3'; IL-8 anti-sense primer, $5^{\prime}$-CAGTGGGGTCCACTCTCAAT- ${ }^{\prime} ; \beta$-actin sense primer, $5^{\prime}$ TG TTCGAGACCTTCAACACG- $3^{\prime} ; \beta$-actin anti-sense primer, $5^{\prime}$-ATCCCCAGAGTCCATGACAA- $3^{\prime}$.

\section{Enzyme-Linked Immunosorbent Assay (ELISA)}

IPEC-J2 cells were seeded in 96-well culture plates and grown until fully confluent. After simultaneous treatment with indicated stimuli for $24 \mathrm{~h}$, the culture media were collected and IL-8 was determined using a porcine IL-8 ELISA kit (R\&D Systems, Minneapolis, MN, United States) according to the manufacturer's instruction.

\section{Western Blot}

IPEC-J2 cells were seeded in 6-well culture plates and grown until fully confluent. After simultaneous treatment with indicated stimuli for $30 \mathrm{~min}$, the cells were lysed in a lysis buffer. Equal amounts of protein were resolved on 10\% SDS-PAGE gels and electro-transferred onto PVDF membranes (Millipore, Bedford, MA, United States). After blocking with 5\% bovine serum albumin, membranes were probed with primary antibodies specific for p38 kinase, phospho-p38 kinase, ERK, phospho-ERK, I $\mathrm{B} \alpha$, or $\beta$-actin (Cell Signaling Technology). After washing, membranes were incubated with HRP-conjugated anti-rabbit IgG as secondary antibody. Immunoreactive bands were detected using a luminescent image analyzer (Fuji Film, Tokyo, Japan).

\section{Statistical Analysis}

Statistical significance was measured using Student's $t$-test and differences were considered significant when $P<0.05$ by
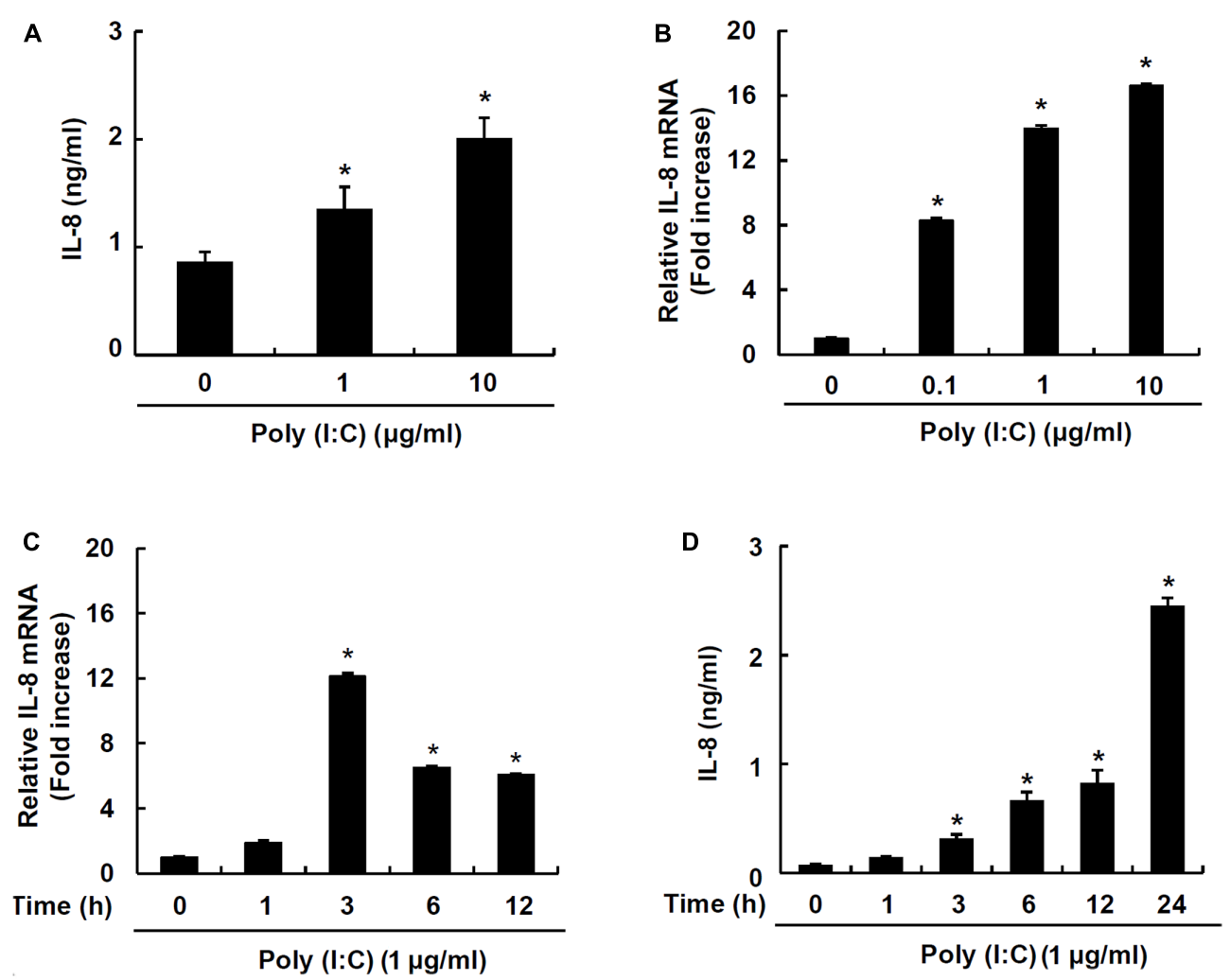

FIGURE 1 | TLR3 ligand poly I:C induces IL-8 production in IPEC-J2 cells. IPEC-J2 cells (5 × $10^{5}$ cells/ml) were plated in 96 -well plates and treated with 1 or $10 \mu \mathrm{g} / \mathrm{ml}$ poly I:C (A) for $24 \mathrm{~h}$ in serum-free medium. Then, the supernatants were collected and IL- 8 secretion was determined by ELISA. IPEC-J2 cells $\left(5 \times 10^{5}\right.$ cells $/ \mathrm{ml}$ ) were plated in 12-well plates and treated with $0.1,1$, or $10 \mu \mathrm{g} / \mathrm{ml} \mathrm{poly} \mathrm{I:C} \mathrm{for} 3 \mathrm{~h}$ (B), or $1 \mu \mathrm{g} / \mathrm{ml} \mathrm{poly} \mathrm{I:C} \mathrm{for} 0,1,3,6$, or $12 \mathrm{~h}$ in serum-free medium (C). After treatment, total RNA was extracted and IL-8 mRNA expression was determined by real-time PCR. Data are mean \pm standard deviation of triplicate samples. (D) IPEC-J2 cells $\left(5 \times 10^{5}\right.$ cells/ml) were plated in 96-well culture plates and treated with $1 \mu \mathrm{g} / \mathrm{ml} \mathrm{poly} \mathrm{I:C} \mathrm{for} \mathrm{1,} \mathrm{3,} \mathrm{6,} 12$ and $24 \mathrm{~h}$, and IL-8 secretion was determined by ELISA. Data are expressed as mean value \pm standard deviation of triplicate samples. Asterisk (*) indicates significant difference compared with the non-treatment control group $(P<0.05)$. 
comparing the experimental group with appropriate control. The data represented one of three independent experiments unless otherwise stated.

\section{RESULTS}

\section{Poly I:C Induces IL-8 Production in IPEC-J2 Cells}

IPEC-J2 has been characterized and commonly used as an in vitro model for studying porcine intestinal pathogen-host interactions
(Botic et al., 2007). To examine whether a synthetic analog of viral double-stranded RNA, poly I:C, induces chemokine production, IPEC-J2 cells were stimulated with poly I:C. Poly I:C significantly increased IL- 8 secretion at 1 and $10 \mu \mathrm{g} / \mathrm{ml}$ poly I:C (Figure 1A). Next, mRNA expression of IL-8 was further examined in IPEC-J2 cells stimulated with $0.1,1$, or $10 \mu \mathrm{g} / \mathrm{ml}$ poly I:C for 3 h. IL-8 mRNA expression was significantly up-regulated at $0.1 \mu \mathrm{g} / \mathrm{ml}$ up to $10 \mu \mathrm{g} / \mathrm{ml}$ poly I:C (Figure 1B). IL-8 mRNA expression peaked at $3 \mathrm{~h}$-treatment (Figure 1C) and IL-8 protein secretion was dose-dependently increased up to $24 \mathrm{~h}$-treatment (Figure 1D).
A

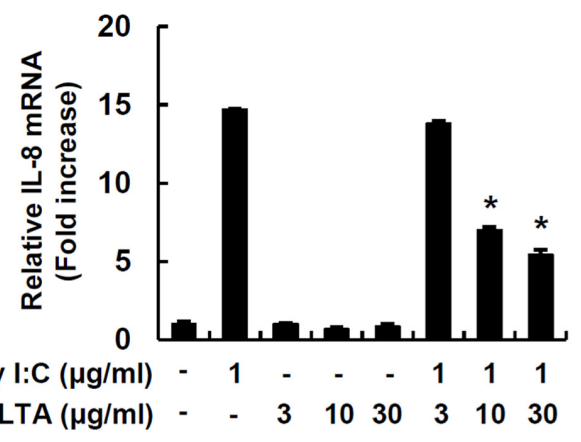

B

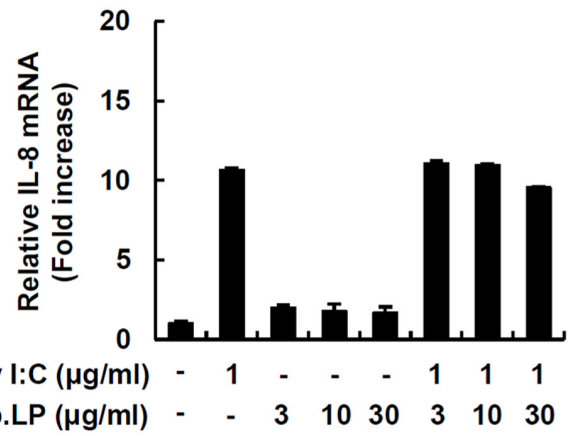

C

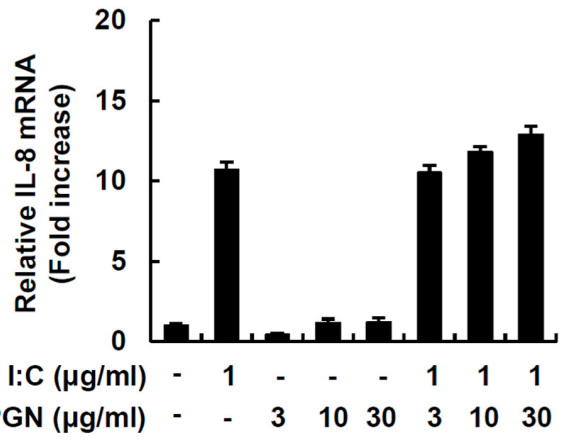

D

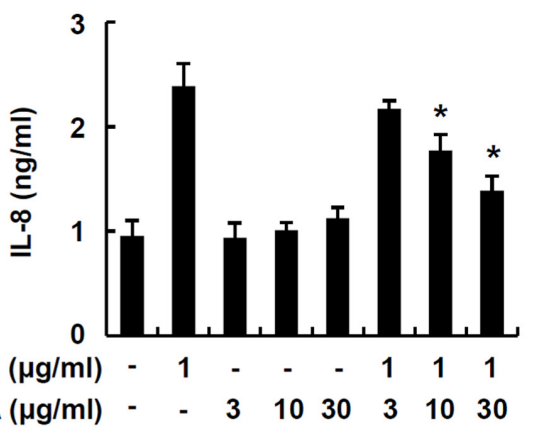

E

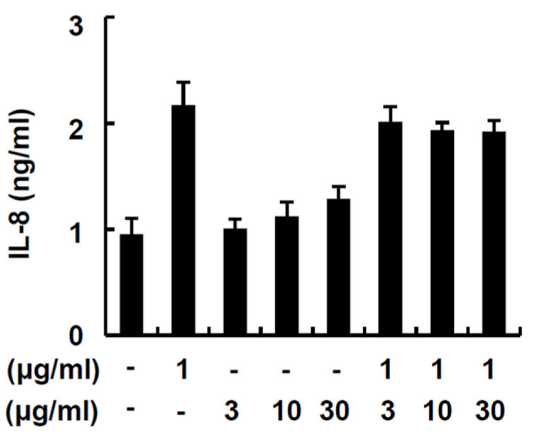

$\mathbf{F}$

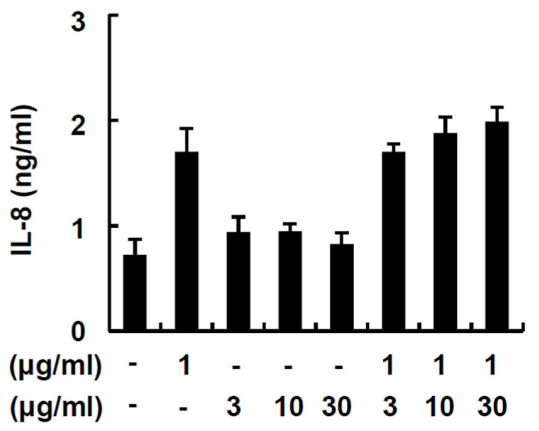

FIGURE 2 | Lp.LTA inhibits poly I:C-induced IL-8 production in IPEC-J2 cells. IPEC-J2 cells $\left(5 \times 10^{5} \mathrm{cells} / \mathrm{ml}\right)$ were plated in 12 -well culture plates and co-treated with poly l:C (1 $\mu \mathrm{g} / \mathrm{ml})$ and Lp.LTA (3-30 $\mu \mathrm{g} / \mathrm{ml})$ (A), Lp.LP (3-30 $\mu \mathrm{g} / \mathrm{ml})$ (B), or Lp.PGN (3-30 $\mu \mathrm{g} / \mathrm{ml})$ (C) for $3 \mathrm{~h}$ in serum-free medium. Then, total RNA was extracted and IL-8 mRNA expression was determined by real-time PCR. Data are mean \pm standard deviation of triplicate samples. Cells were co-treated with poly I:C (1 $\mu \mathrm{g} / \mathrm{ml})$ and Lp.LTA (3-30 $\mu \mathrm{g} / \mathrm{ml})$ (D), Lp.LP (3-30 $\mu \mathrm{g} / \mathrm{ml})$ (E), or Lp.PGN (3-30 $\mu \mathrm{g} / \mathrm{ml})(\mathbf{F})$ for $24 \mathrm{~h}$ to examine the supernatant for IL-8 protein using ELISA. Data are mean \pm standard deviation of triplicate samples. Asterisk $\left(^{*}\right)$ indicates a significant difference compared with poly I:C-treated control group $(P<0.05)$. 


\section{Lp.LTA Exclusively Inhibits Poly I:C-Induced IL-8 Production in IPEC-J2 Cells}

In order to investigate whether major cell wall components were responsible for the inhibitory effect on porcine intestinal inflammatory responses, IPEC-J2 cells were treated with LTA, lipoproteins or PGN from L. plantarum in the presence of poly I:C. Only Lp.LTA inhibited IL-8 mRNA expression at 10 and $30 \mu \mathrm{g} / \mathrm{ml}$. However, lipoproteins of L. plantarum (Lp.LP) or PGN of $L$. plantarum (Lp.PGN) did not inhibit poly I:C-induced IL-8 mRNA expression (Figures 2A-C). Similar to the results for mRNA, Lp.LTA, but not Lp.LP or Lp.PGN, significantly inhibited poly I:C-induced IL-8 secretion at 10 and $30 \mu \mathrm{g} / \mathrm{ml}$ (Figures 2D-F).

\section{Lactobacillus LTAs Do Not Commonly Inhibit Poly I:C-Induced IL-8 Production in IPEC-J2 Cells}

To further examine whether other probiotic Lactobacillus LTAs inhibit poly I:C-induced IL-8 production, IPEC-J2 cells were treated with poly I:C and various Lactobacillus LTAs. Figure 3 displayed that Lp.LTA and LTA from L. delbrueckii (Ld.LTA) significantly inhibited poly I:C-induced IL-8 secretion at $30 \mu \mathrm{g} / \mathrm{ml}$. However, LTAs from L. sakei (Ls.LTA) and L. rhamnosus GG (Lr.LTA) did not inhibit poly I:C-induced IL-8 secretion, suggesting that other Lactobacillus LTAs did not commonly inhibit poly I:C-induced IL-8 production in IPEC-J2 cells.

\section{D-Alanine and Lipid Moieties of Lp.LTA Are Critical for Inhibition of Poly I:C-Induced IL-8 Production in IPEC-J2 Cells}

In order to determine whether the D-alanine moiety of Lp.LTA was involved in the inhibition of poly I:C-induced IL-8 production, IPEC-J2 cells were treated with dealanylated Lp.LTA in the presence of poly I:C. Similar to results with native Lp.LTA, dealanylated Lp.LTA alone did not induce IL-8 secretion in IPECJ2 cells. Although native Lp.LTA significantly inhibited poly I:C-induced IL-8 secretion, dealanylated Lp.LTA failed to inhibit poly I:C-induced IL-8 secretion (Figure 4). Next, we examined the inhibitory potential of deacylated Lp.LTA. Deacylated Lp.LTA did not inhibit poly I:C-induced IL-8 secretion (Figure 4). These results indicated that $\mathrm{D}$-alanine and lipid moieties of Lp.LTA were involved in the inhibition of poly I:C-induced IL- 8 production in IPEC-J2 cells.

\section{Lp. LTA Decreases Poly I:C-Induced MAPK Phosphorylation and NF- $\kappa B$} Activation

IPEC-J2 cells were treated with poly I:C and/or Lp.LTA to determine MAPK phosphorylation and NF- $\kappa$ B activation. Poly I:C alone enhanced ERK and p38 phosphorylation, but the presence of Lp.LTA significantly decreased poly I:C-mediated
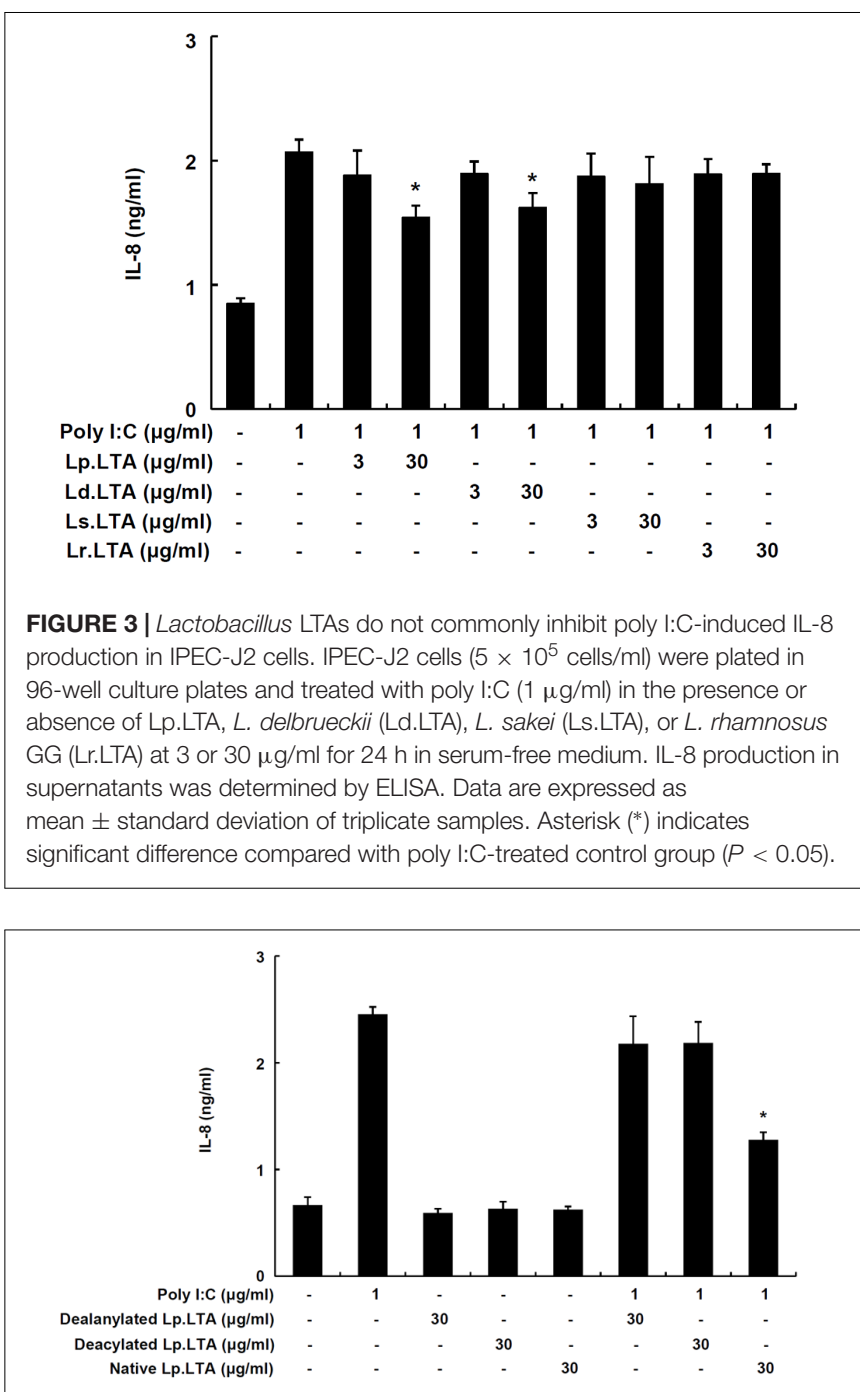

FIGURE 4 | D-Alanine and lipid moieties of Lp.LTA are essential for inhibition of poly I:C-induced IL-8 production in IPEC-J2 cells. IPEC-J2 cells $\left(5 \times 10^{5}\right.$ cells $/ \mathrm{ml}$ ) were plated in 96-well culture plates and treated with poly I:C $(1 \mu \mathrm{g} / \mathrm{ml})$ in the presence or absence of dealanyated Lp.LTA, deacylated Lp.LTA or native Lp.LTA (all $30 \mu \mathrm{g} / \mathrm{ml}$ ) for $24 \mathrm{~h}$. And then, IL-8 secretion in the supernatant was determined by ELISA. Data are expressed as mean \pm standard deviation of triplicate samples. Asterisk $(*)$ indicates significant difference compared with appropriate control $(P<0.05)$.

phosphorylation of ERK and p38 (Figures 5A-C). Moreover, poly I:C alone induced $\mathrm{I} \kappa \mathrm{B} \alpha$ degradation, leading to NF- $\kappa \mathrm{B}$ activation, whereas Lp.LTA blocked poly I:C-induced IкB $\alpha$ degradation, suggesting that Lp.LTA reduced NF- $\kappa$ B activation (Figures 5A,D). It is likely that Lp.LTA regulated MAPK phosphorylation and NF- $\kappa \mathrm{B}$ activation, leading to the reduction of poly I:C-induced IL-8 production in IPEC-J2.

\section{DISCUSSION}

In the livestock industry, probiotics are used in piglets to improve growth and digestibility, prevent diarrhea, and modulate immune 


\section{A}

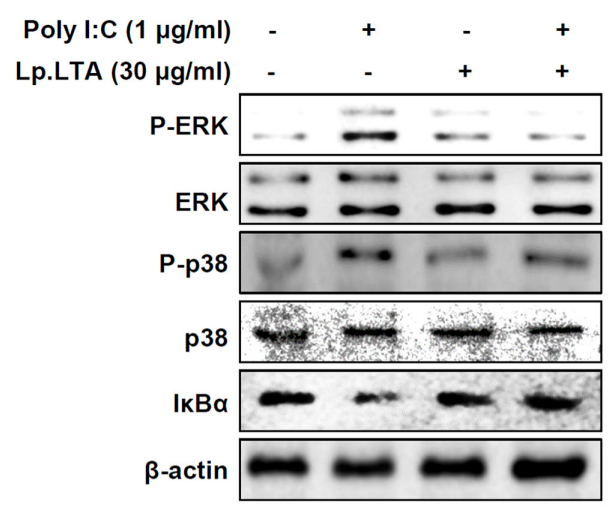

C

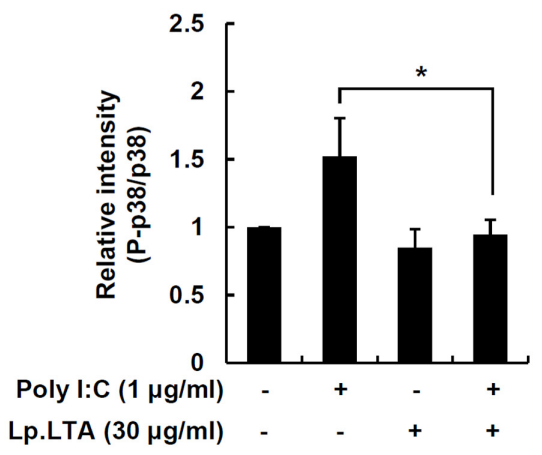

B

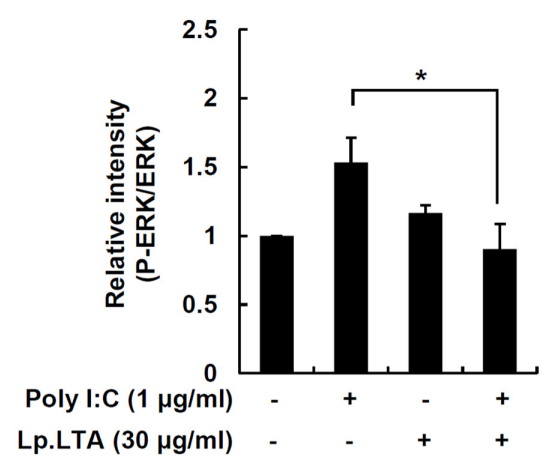

D

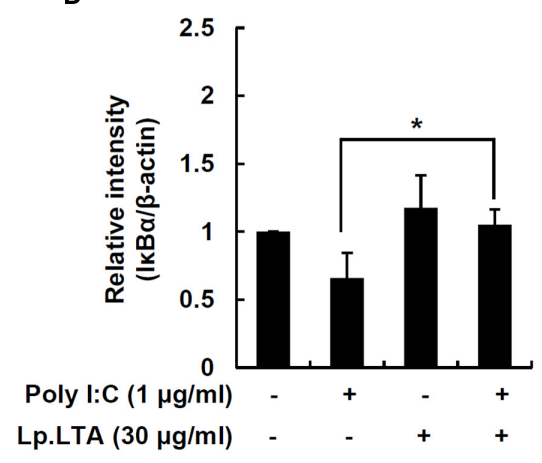

FIGURE 5 | Lp.LTA reduces poly I:C-induced activation of p38 kinase, ERK and NF-kB in IPEC-J2 cells. IPEC-J2 cells (5 × $10^{5}$ cells/ml) were grown in serum-free medium overnight. Cells were treated with poly I:C (1 $\mu \mathrm{g} / \mathrm{ml})$ in the presence or absence of Lp.LTA (30 $\mu \mathrm{g} / \mathrm{ml})$ for 30 min. Cells were lysed and equal amounts of proteins were analyzed by Western blots using antibodies specific to phosphorylated or non-phosphorylated ERK, p38 kinase or IкB $\alpha$ (A). The intensity of each band was determined using an imaging densitometer and relative protein level calculated based on intensity of non-phosphorylated ERK (B), non-phosphorylated p38 (C), or $\beta$-actin (D). Data are expressed as mean \pm standard deviation from three independent experiments. Asterisk $(*)$ indicates significant difference compared with appropriate control $(P<0.05)$.

responses in the gut (Hou et al., 2015). A number of pathogenic bacteria and several viruses including group A rotavirus are associated with acute diarrhea in weaning and post-weaning piglets (Martella et al., 2010). Although the use of probiotics is widely accepted as an alternative to antibiotics, the effector molecules of probiotic bacteria responsible for the prevention of virus-induced diarrhea in piglets have not been identified. In this study, we demonstrated that a major cell wall component, LTA of probiotic L. plantarum, inhibited a synthetic analog of double-stranded RNA derived from viruses, poly I:C-induced IL-8 production in porcine intestinal epithelial cells.

Unlike LTA from pathogenic bacteria, LTA from probiotic bacteria such as Lactobacillus distinctively acts as an antiinflammatory substance. For example, Lp.LTA suppressed a synthetic lipopeptide, Pam2CSK4-induced IL-8 production in human intestinal epithelial cells (Noh et al., 2015). LPS-induced endotoxin shock was reduced by the administration with Lp.LTA in a mouse model, which up-regulated IL-1R-associated kinase$\mathrm{M}$, a negative regulator, to lessen excessive inflammatory responses (Kim et al., 2008). In accordance with previous observations, Lp.LTA effectively inhibited TLR3-mediated inflammatory responses in porcine intestinal epithelial cells. It is likely that Lp.LTA may have broad spectrum of inhibitory actions on bacterial and viral inflammatory responses. In addition, D-alanine and lipid moieties of LTA are involved in immunostimulating potential (Ryu et al., 2009). Deficiency of D-alanine or lipid moieties of Lp.LTA completely lost the inhibitory effect on IL- 8 production, suggesting that $\mathrm{D}$-alanine and lipid moieties of Lp.LTA are essential components in the inhibition of poly I:C-induced IL-8 production in porcine intestinal epithelial cells.

TLR2 is known to associate with the pathogenesis of microbial infections (Kang et al., 2016). It is also important for host protection against infection. For instance, Lp.LTA attenuated MAPK phosphorylation and NF- $\kappa \mathrm{B}$ activation by reducing TLR2 activation, resulting in an anti-inflammatory response in human intestinal epithelial cells (Noh et al., 2015). This observation is similar to our study, in which Lp.LTA as a 
TLR2 ligand regulated MAPK phosphorylation and NF- $\kappa$ B activation, presuming that interaction of Lp.LTA with TLR2 was involved in the anti-inflammatory response. In contrast to our finding, TLR2 combined with TLR3 enhances the induction of inflammatory cytokines such as tumor necrosis factor (TNF)- $\alpha$ and IL- 6 , but inhibits IFN-stimulated genes such as IFN- $\beta$ in myeloid dendritic cells (Vanhoutte et al., 2008). However, among TLR2 ligands such as LTA, PGN, and synthetic lipopeptides, LTA from Staphylococcus epidermis and $S$. aureus suppressed poly I:C-induced TNF- $\alpha$, blocking the activation of $\mathrm{NF}-\kappa \mathrm{B}$ by up-regulating the negative regulatory molecule TNF receptor-associated factor-1 (Lai et al., 2009). These results suggest that a negative regulatory mechanism(s) might be involved in the inhibitory effect of Lp.LTA on IL- 8 production in porcine intestinal epithelial cells.

Growing evidence supports the therapeutic and preventive applications of probiotics against gastrointestinal inflammatory reactivity in animals and humans. Of note, probiotic effects are often species- or strain-specific (Lee et al., 2013). We demonstrated that Lp.LTA contributed to the anti-inflammatory responses in porcine intestines. Our results provide an understanding of the interaction between a probiotic effector molecule and intestinal epithelial cells for the prevention or treatment of gut inflammatory disorders in the livestock. This study clearly showed that LTA as a probiotic effector molecule contributed to the probiotic interaction with porcine

\section{REFERENCES}

Agustina, R., Kok, F. J., Van De Rest, O., Fahmida, U., Firmansyah, A., Lukito, W., et al. (2012). Randomized trial of probiotics and calcium on diarrhea and respiratory tract infections in Indonesian children. Pediatrics 129, e1155-e1164. doi: $10.1542 /$ peds.2011-1379

Bermudez-Brito, M., Plaza-Diaz, J., Munoz-Quezada, S., Gomez-Llorente, C., and Gil, A. (2012). Probiotic mechanisms of action. Ann. Nutr. Metab. 61, 160-174. doi: 10.1159/000342079

Botic, T., Klingberg, T. D., Weingartl, H., and Cencic, A. (2007). A novel eukaryotic cell culture model to study antiviral activity of potential probiotic bacteria. Int. J. Food Microbiol. 115, 227-234. doi: 10.1016/j.ijfoodmicro.2006.10.044

Bron, P. A., Tomita, S., Mercenier, A., and Kleerebezem, M. (2013). Cell surfaceassociated compounds of probiotic lactobacilli sustain the strain-specificity dogma. Curr. Opin. Microbiol. 16, 262-269. doi: 10.1016/j.mib.2013.06.001

Donato, K. A., Gareau, M. G., Wang, Y. J., and Sherman, P. M. (2010). Lactobacillus rhamnosus GG attenuates interferon-\{gamma\} and tumour necrosis factor-alpha-induced barrier dysfunction and pro-inflammatory signalling. Microbiology 156, 3288-3297. doi: 10.1099/mic.0.040139-0

Grangette, C., Nutten, S., Palumbo, E., Morath, S., Hermann, C., Dewulf, J., et al. (2005). Enhanced antiinflammatory capacity of a Lactobacillus plantarum mutant synthesizing modified teichoic acids. Proc. Natl. Acad. Sci. U.S.A. 102, 10321-10326. doi: 10.1073/pnas.0504084102

Hou, C., Zeng, X., Yang, F., Liu, H., and Qiao, S. (2015). Study and use of the probiotic Lactobacillus reuteri in pigs: a review. J. Anim. Sci. Biotechnol. 6:14. doi: 10.1186/s40104-015-0014-3

Kailasapathy, K., and Chin, J. (2000). Survival and therapeutic potential of probiotic organisms with reference to Lactobacillus acidophilus and Bifidobacterium spp. Immunol. Cell Biol. 78, 80-88. doi: 10.1046/j.1440-1711. 2000.00886.x

Kang, S. S., Noh, S. Y., Park, O. J., Yun, C. H., and Han, S. H. (2015). Staphylococcus aureus induces IL-8 expression through its lipoproteins in the human intestinal epithelial cell, Caco-2. Cytokine 75, 174-180. doi: 10.1016/j.cyto.2015.04.017 intestinal epithelial cells that is feasible to be used in animal feeding.

\section{AUTHOR CONTRIBUTIONS}

S-SK, C-HY, and SHH conceived the research. KWK, S-JW, and $\mathrm{S}$-SK performed the experiments. S-SK, C-HY, and SHH wrote the manuscript. O-JP, K-DS, KBA, and H-KL interpreted data and edited manuscript. All authors reviewed and accepted the manuscript.

\section{ACKNOWLEDGMENTS}

This work was supported by grants from the National Research Foundation of Korea, which is funded by the Korean government (NRF-2015R1A2A1A15055453, NRF-2015M2A2A6A01044894, and NRF-2015R1D1A1A09056592), Next-Generation BioGreen 21 Program (PJ01112402), Rural Development Administration, and the Korea Health Technology R\&D Project through the Korea Health Industry Development Institute (KHIDI), funded by the Ministry of Health \& Welfare (HI17C1377), South Korea. This research was also a part of the project titled 'Development of global senior-friendly health functional food materials from marine resources,' funded by the Ministry of Oceans and Fisheries, South Korea.

Kang, S. S., Sim, J. R., Yun, C. H., and Han, S. H. (2016). Lipoteichoic acids as a major virulence factor causing inflammatory responses via Toll-like receptor 2. Arch. Pharm. Res. 39, 1519-1529. doi: 10.1007/s12272-016-0804-y

Kim, H. G., Kim, N. R., Gim, M. G., Lee, J. M., Lee, S. Y., Ko, M. Y., et al. (2008). Lipoteichoic acid isolated from Lactobacillus plantarum inhibits lipopolysaccharide-induced TNF-alpha production in THP-1 cells and endotoxin shock in mice. J. Immunol. 180, 2553-2561. doi: 10.4049/jimmunol. 180.4.2553

Kopp-Hoolihan, L. (2001). Prophylactic and therapeutic uses of probiotics: a review. J. Am. Diet. Assoc. 101, 229-238. doi: 10.1016/S0002-8223(01)00060-8

Lai, Y., Di Nardo, A., Nakatsuji, T., Leichtle, A., Yang, Y., Cogen, A. L., et al. (2009). Commensal bacteria regulate Toll-like receptor 3-dependent inflammation after skin injury. Nat. Med. 15, 1377-1382. doi: 10.1038/nm.2062

Lee, I. C., Tomita, S., Kleerebezem, M., and Bron, P. A. (2013). The quest for probiotic effector molecules-unraveling strain specificity at the molecular level. Pharmacol. Res. 69, 61-74. doi: 10.1016/j.phrs.2012.09.010

Lievin-Le Moal, V., and Servin, A. L. (2014). Anti-infective activities of lactobacillus strains in the human intestinal microbiota: from probiotics to gastrointestinal anti-infectious biotherapeutic agents. Clin. Microbiol. Rev. 27, 167-199. doi: 10.1128/CMR.00080-13

Macho Fernandez, E., Valenti, V., Rockel, C., Hermann, C., Pot, B., Boneca, I. G., et al. (2011). Anti-inflammatory capacity of selected lactobacilli in experimental colitis is driven by NOD2-mediated recognition of a specific peptidoglycanderived muropeptide. Gut 60, 1050-1059. doi: 10.1136/gut.2010.232918

Martella, V., Banyai, K., Matthijnssens, J., Buonavoglia, C., and Ciarlet, M. (2010). Zoonotic aspects of rotaviruses. Vet. Microbiol. 140, 246-255. doi: 10.1016/j. vetmic.2009.08.028

Morath, S., Geyer, A., and Hartung, T. (2001). Structure-function relationship of cytokine induction by lipoteichoic acid from Staphylococcus aureus. J. Exp. Med. 193, 393-397. doi: 10.1084/jem.193.3.393

Morath, S., Stadelmaier, A., Geyer, A., Schmidt, R. R., and Hartung, T. (2002). Synthetic lipoteichoic acid from Staphylococcus aureus is a potent stimulus of cytokine release. J. Exp. Med. 195, 1635-1640. doi: 10.1084/jem.20020322 
Noh, S. Y., Kang, S. S., Yun, C. H., and Han, S. H. (2015). Lipoteichoic acid from Lactobacillus plantarum inhibits Pam2CSK4-induced IL-8 production in human intestinal epithelial cells. Mol. Immunol. 64, 183-189. doi: 10.1016/j. molimm.2014.11.014

O'Shea, E. F., Cotter, P. D., Stanton, C., Ross, R. P., and Hill, C. (2012). Production of bioactive substances by intestinal bacteria as a basis for explaining probiotic mechanisms: bacteriocins and conjugated linoleic acid. Int. J. Food Microbiol. 152, 189-205. doi: 10.1016/j.ijfoodmicro.2011.05.025

Rolfe, R. D. (2000). The role of probiotic cultures in the control of gastrointestinal health. J. Nutr. 130, 396S-402S.

Ryu, Y. H., Baik, J. E., Yang, J. S., Kang, S. S., Im, J., Yun, C. H., et al. (2009). Differential immunostimulatory effects of Gram-positive bacteria due to their lipoteichoic acids. Int. Immunopharmacol. 9, 127-133. doi: 10.1016/j.intimp. 2008.10.014

Schrezenmeir, J., and de Vrese, M. (2001). Probiotics, prebiotics, and synbioticsapproaching a definition. Am. J. Clin. Nutr. 73, 361S-364S.

Snel, J., Vissers, Y. M., Smit, B. A., Jongen, J. M., Van Der Meulen, E. T., Zwijsen, R., et al. (2011). Strain-specific immunomodulatory effects of Lactobacillus plantarum strains on birch-pollen-allergic subjects out of season. Clin. Exp. Allergy 41, 232-242. doi: 10.1111/j.1365-2222.2010.03650.x

Thacker, P. A. (2013). Alternatives to antibiotics as growth promoters for use in swine production: a review. J. Anim. Sci. Biotechnol. 4:35. doi: 10.1186/20491891-4-35

Thomas, C. M., and Versalovic, J. (2010). Probiotics-host communication: modulation of signaling pathways in the intestine. Gut Microbes 1, 148-163. doi: $10.4161 /$ gmic. 1.3 .11712 van der Fels-Klerx, H. J., Puister-Jansen, L. F., Van Asselt, E. D., and Burgers, S. L. (2011). Farm factors associated with the use of antibiotics in pig production. J. Anim. Sci. 89, 1922-1929. doi: 10.2527/jas. 2010-3046

Vanhoutte, F., Paget, C., Breuilh, L., Fontaine, J., Vendeville, C., Goriely, S., et al. (2008). Toll-like receptor (TLR)2 and TLR3 synergy and cross-inhibition in murine myeloid dendritic cells. Immunol. Lett. 116, 86-94. doi: 10.1016/j.imlet. 2007.11.014

Villena, J., and Kitazawa, H. (2014). Modulation of intestinal TLR4-inflammatory signaling pathways by probiotic microorganisms: lessons learned from Lactobacillus jensenii TL2937. Front. Immunol. 4:512. doi: 10.3389/fimmu.2013. 00512

Yang, F., Hou, C., Zeng, X., and Qiao, S. (2015). The use of lactic acid bacteria as a probiotic in swine diets. Pathogens 4, 34-45. doi: 10.3390/pathogens4010034

Conflict of Interest Statement: The authors declare that the research was conducted in the absence of any commercial or financial relationships that could be construed as a potential conflict of interest.

Copyright (c) 2017 Kim, Kang, Woo, Park, Ahn, Song, Lee, Yun and Han. This is an open-access article distributed under the terms of the Creative Commons Attribution License (CC BY). The use, distribution or reproduction in other forums is permitted, provided the original author(s) or licensor are credited and that the original publication in this journal is cited, in accordance with accepted academic practice. No use, distribution or reproduction is permitted which does not comply with these terms. 